\title{
Vickers micro-indentation for the elasto-plastic characterisation of thin layers
}

\author{
S. Syngellakis ${ }^{1}$ \& O. C. Summerfield ${ }^{2}$ \\ ${ }^{1}$ Wessex Institute of Technology, UK \\ ${ }^{2}$ University of Southampton, UK
}

\begin{abstract}
A method is proposed for characterising components whose geometry rules out traditional tensile testing as a means of determining their elasto-plastic properties, especially if the components' manufacturing process is expected to have a significant influence on these properties. Suitable sets of material parameters are identified that fully characterise both the elasto-plastic and indentation behaviour of tested aluminium alloys. Based on a series of finite element simulations of Vickers indentations, a surrogate model is built to map the elasto-plastic parameters to indentation parameters for a wide range of alloy behaviour. The genetic algorithm is then employed to solve the inverse problem, that is, to work backwards from indentation to elasto-plastic parameters, allowing predictions of stress-strain curves from experimental indentation data. The developed characterisation process is shown to be capable of predicting quite well the general trends in an alloy's elasto-plastic behaviour. Scope for further work, which could improve the results, is identified.

Keywords: Vickers hardness, aluminium alloys, elasto-plastic behaviour, surrogate modelling.
\end{abstract}

\section{Introduction}

The elasto-plastic properties are essential input to the analysis of material behaviour for the assessment of possible failure mechanisms in machine or structural components. In the case of thin material layers, such as those encountered in automotive plain bearings, material properties originating from experiments on bulk materials are severely modified by the manufacturing process. Due to the difficulty in applying traditional testing techniques to such 
thin and often curved layers, hardness indentation has been proposed and investigated as a feasible alternative. There have been numerous experimental and analytical studies on the effectiveness and accuracy of this approach. The common characteristic of most such studies is the attempt at a direct link between indentation output and elasto-plastic properties based on purely solid mechanics considerations. Tabor's classical work [1] has been the basis and inspiration of this approach whose scope has been significantly expanded through the systematic use of finite element (FE) modelling [2, 3]; the latter has more recently been combined with load-indentation data from instrumented nano-hardness tests [4].

Surrogate modelling or meta-modelling techniques have been applied to material characterisation as a means of interpolating between experimental or FE-simulated data and thus obtaining optimum correlation between these data and material properties with considerably reduced computational effort [5]. Such approaches for predicting elasto-plastic material parameters have been based on results from instrumented nano-indentation tests [6, 7], which are highly demanding in both specimen preparation and equipment.

The aim of the present work is to apply a general methodology for determining elasto-plastic material properties from relatively simple microhardness tests, which allow the measurement of hardness within a few millimetres of material thickness. There has been one such previous attempt [8] based on the assumption that micro-hardness indent size variation with load depends strongly on the elasto-plastic properties of such thin layers. This hypothesis is again tested by providing further experimental evidence of both tensile and indentation behaviour, alternative choices of characterisation parameters and the application of a more versatile surrogate modelling tool, which includes the optimisation routine required for the solution of the inverse problem.

\section{Methodology}

The Vickers micro-hardness testing technique uses a square-based diamond pyramid as an indenter. The actual test data used for elasto-plastic characterisation are the diagonal size of the permanent indentation $\delta$ and the corresponding applied load $P$ so that experimental $P$ - $\delta$ relations can be generated. Rather than being extracted from a rigorous theoretical analysis of the micro-hardness test results, here elasto-plastic information for a new material is identified as that leading to the numerical prediction of the same load-indentation characteristics as the experimental ones. This inverse problem can only be solved through an iterative scheme within an optimization algorithm. A great advantage of this approach is the flexibility in choosing sets of either material or indentation parameters.

The finite element (FE) method can be used to link numerically known elastoplastic stress $(\sigma)-$ strain $(\varepsilon)$ relations with respective indentation output. Since however the repeated FE simulation of indentations required by an optimisation scheme would have been computationally very expensive and made 


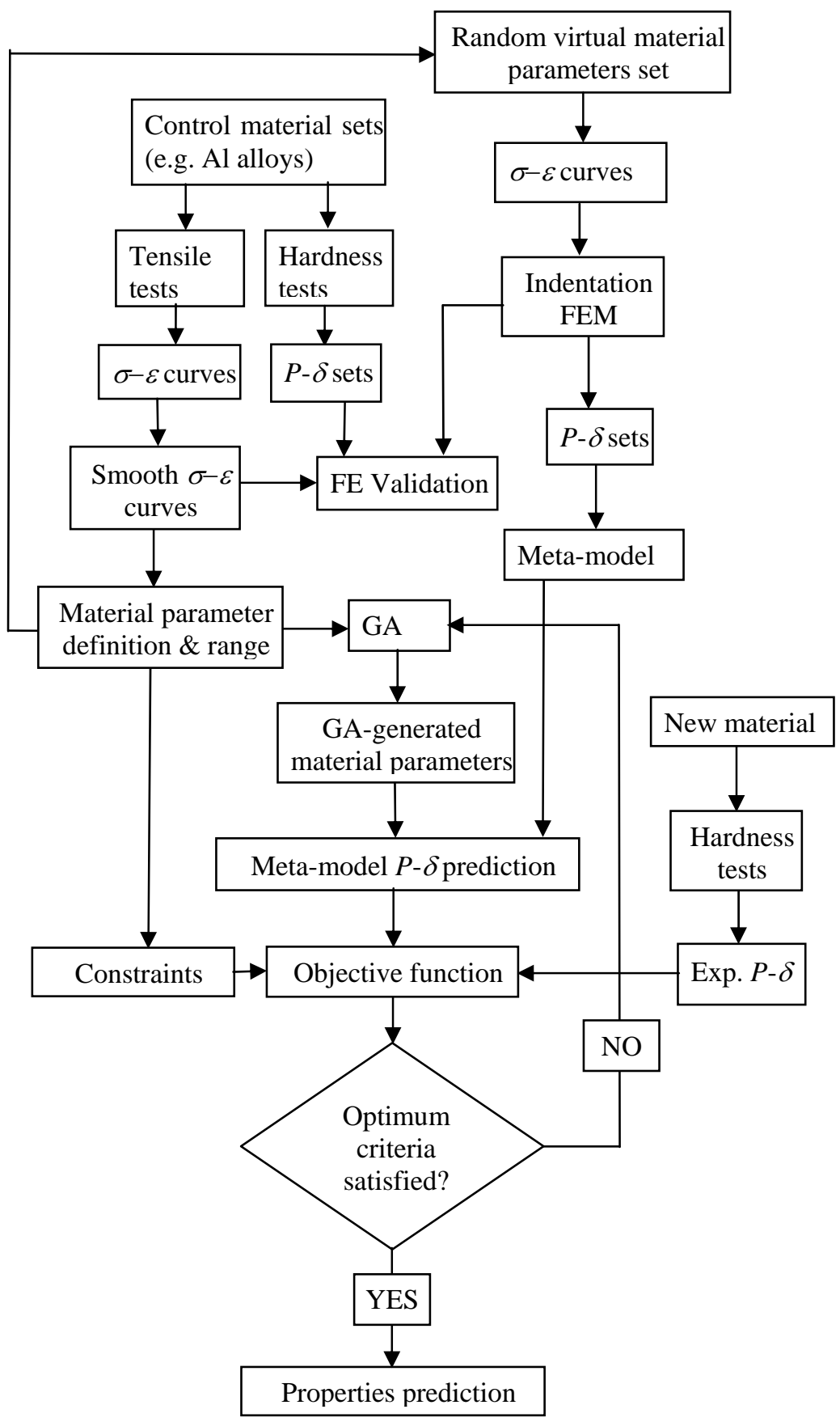

Figure 1: $\quad$ Flow chart of the adopted characterisation process. 
the application of the method practically impossible, surrogate modelling is adopted as an alternative means of correlating a given set of material data with indentation behaviour. Building up a reliable surrogate model requires a substantial amount of data, which can be obtained through either a prolonged experimental programme or multiple FE simulations of the indentation process. The latter is more versatile since it admits any set of material data thus ensuring their uniform distribution within their ranges.

Initially, micro-hardness tests are carried out on a small number of specimens with known, wide-ranging $\sigma-\varepsilon$ characteristics, which provide the key parameters describing as accurately as possible elasto-plastic material behaviour and are used as input to FE indentation analyses performed solely for validation purposes. Once the reliability of the FE predictions is established, an expanded database of $P$ - $\delta$ curves is generated by running the FE model for realistic ranges of the adopted material parameters. This larger database is used for training surrogate modelling schemes approximately relating the indentation to material parameters. Based on iterative application of the most appropriate surrogate model within an optimisation algorithm, the properties of a material with given experimental micro hardness $P$ - $\delta$ curves can be determined. A flowchart of the methodology is shown in fig. 1 .

\section{Experiments}

\subsection{Tensile tests}

Three aluminium alloy grades were tested: $\mathrm{Al} \mathrm{1050,} \mathrm{Al} 8090 \mathrm{~T} 3$ and a general purpose alloy designated GP. Multiple specimens of each grade were prepared according to ASTM standards [9] and the tests were performed on an Instron rig with a constant cross-head velocity of $1 \mathrm{~mm} / \mathrm{min}$. Consistent behaviour was observed between specimens of the same grade. The raw data were initially recorded as load and engineering strain and eventually converted to true stress $(\sigma)$-logarithmic strain $(\varepsilon)$ data. The latter are plotted in fig. 2 together with the data from three more, previously tested aluminium alloys, designated B1Q2, VQ1B and AS1241 [8].

A commonly used model for the $\sigma-\varepsilon$ relation is the equation

$$
\sigma=A \varepsilon^{n}
$$

where $A$ and $n$ are known as the strain hardening coefficient and strain hardening exponent respectively [10]. However, this equation very rarely represents accurately the entire experimental $\sigma-\varepsilon$ curve but rather part of it starting from point $\left(\sigma_{1}, \varepsilon_{1}\right)$ beyond the yield point $\left(\sigma_{Y}, \varepsilon_{Y}\right)$. A second order polynomial can be used to represent the experimental $\sigma-\varepsilon$ relation between these two points with satisfactory accuracy. The coefficients of the fitted polynomial can be expressed in terms of $\sigma_{Y}, \sigma_{1}, A$ and $n$, therefore these four parameters suffice to characterise elasto-plastic behaviour; their values were extracted from the curves of fig. 2 and listed in table 1. 


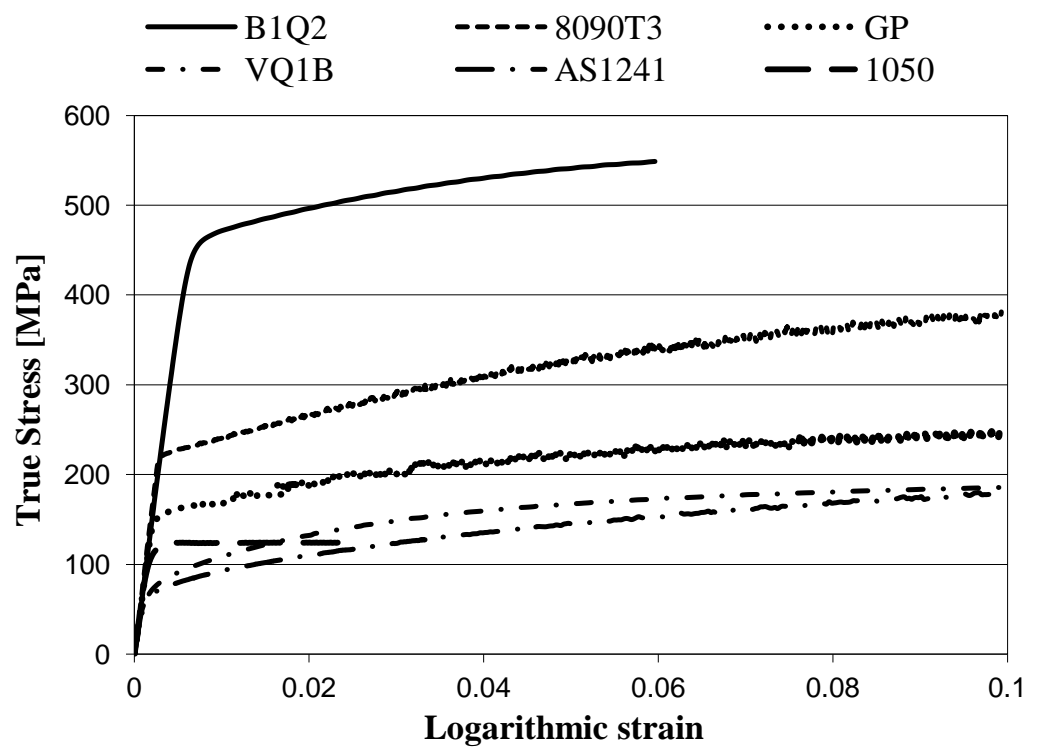

Figure 2: $\quad$ Experimental $\sigma-\varepsilon$ curves for the tested aluminium samples.

Table 1: $\quad$ Elasto-plastic and Vickers hardness characterisation parameters for the tested aluminium samples.

\begin{tabular}{|c|c|c|c|c|c|c|}
\hline Material & $\begin{array}{c}\sigma_{Y} \\
(\mathrm{MPa})\end{array}$ & $\begin{array}{c}\sigma_{1} \\
(\mathrm{MPa})\end{array}$ & $\begin{array}{c}A \\
(\mathrm{MPa})\end{array}$ & $n$ & $B^{(\mathrm{a})}$ & $m$ \\
\hline B1Q2 & 415.1 & 455 & 703.4 & 0.0881 & 0.0369 & 2.1634 \\
\hline 8090 T3 & 217 & 265.3 & 626 & 0.2181 & 0.0552 & 2.0006 \\
\hline GP & 144.9 & 187.5 & 355.5 & 0.1574 & 0.0133 & 2.1595 \\
\hline VQ1B & 39.95 & 87.44 & 390.7 & 0.2767 & 0.0133 & 2.1595 \\
\hline AS1241 & 39.81 & 98.7 & 358.1 & 0.3014 & 0.0190 & 2.0427 \\
\hline 1050 & 66.6 & 124 & 126.3 & 0.0042 & 0.0214 & 1.9842 \\
\hline
\end{tabular}

\subsection{Vickers tests}

Specimens were prepared from the same three aluminium alloys according to the relevant ASTM standards [9]. The samples were mounted on to a thermosetting plastic and then their surface was smoothed to improve the definition of the indentation edges. The load $P$ was applied through the standard diamond squarebased pyramid Vickers indenter for a period of $20 \mathrm{~s}$ and the indentation diagonal $\delta$ was measured to within $\pm 0.5 \mu \mathrm{m}$. Five indentations were made at each load. 
The experimentally obtained $P-\delta$ relations are plotted in fig. 3. Data from the previously tested aluminium alloys, B1Q2, VQ1B and AS1241 [8], are also included in fig. 3. Since the motivation for the development of this methodology was the characterisation of aluminium linings in automotive plain bearings, a typical aluminium alloy used for this purpose was also tested. Samples were supplied by the manufacturer for a bearing design designated MAS16 in three forms: aluminium sheet before bearing manufacturing, multi-layer flat bar and formed bearing half-shell. The data from these three samples show clearly the significant effect of the manufacturing process on the elasto-plastic properties of the aluminium lining. Collectively, the data of fig. 3 provide a good indication of the characterisation range.

All experimental Vickers data were fitted to the empirical relation

$$
P=B \delta^{m}
$$

which is a variation of Meyer's law. This approximation proved to be very good with the fitted curves having a regression coefficient $R^{2}>0.999$. Thus, parameters $B$ and $m$ were assumed to characterise fully the load-indentation curve and were adopted as the independent variables of the developed surrogate model.

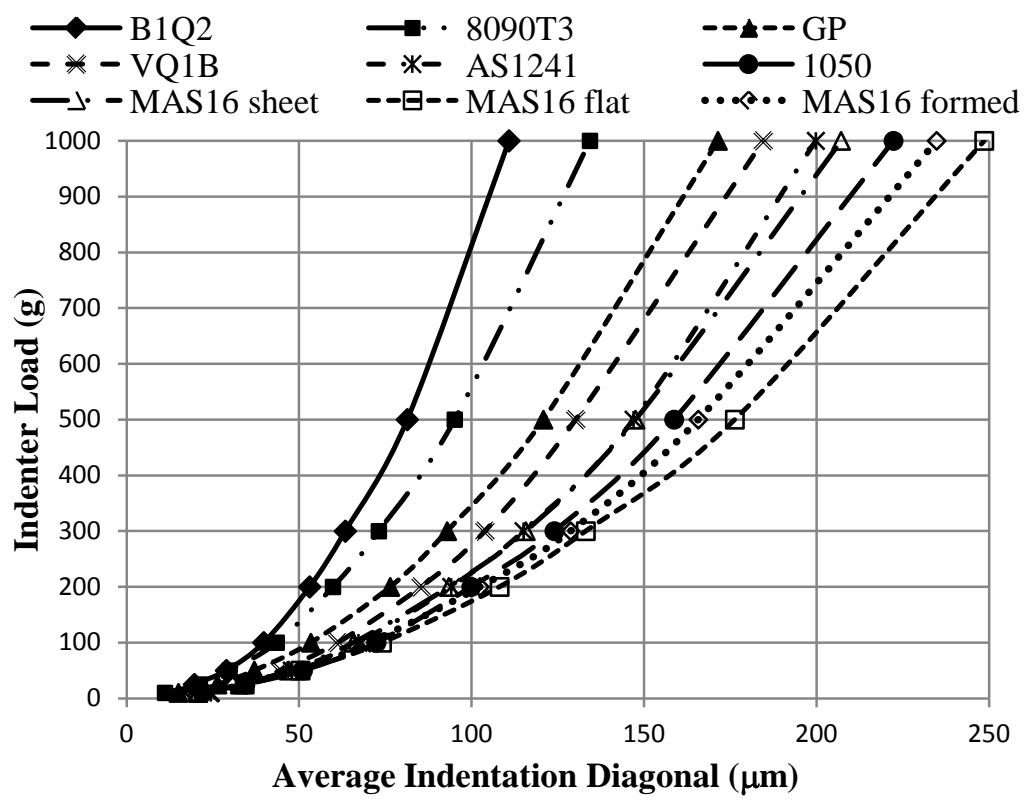

Figure 3: Vickers indentation data. 


\section{Characterisation}

\subsection{Finite element modelling}

Vickers indentations were simulated using the FE model developed in the context of the previous characterisation project at Southampton [8]. This axisymmetric model was validated by comparing its predictions with those obtained from a three-dimensional simulation of the equivalent pyramid indentation as well as experimental indentation data. The model's sensitivity to mesh refinement, far-field boundary conditions and various contact modelling parameters was thoroughly investigated.

The program was again validated using the experimental data from section 3. Two $\sigma-\varepsilon$ inputs were used: one comprising the experimental data, adjusted to constitute a smooth curve with a constantly decreasing gradient and another recreated by the curve fitting process described in section 3.1 using the characterisation parameters of table 1 . The Young's modulus $E$ extracted from the tensile test data was approximately equal to $70 \mathrm{GPa}$ and this value was adopted in all FE simulations of Vickers indentations. It is expected that the indentation predictions would be insensitive to Young's modulus variations since elastic deformation was removed from both the experimental and numerical $P-\delta$ records.

The agreement of FE predictions of indentation behaviour, for both sampled and fitted stress-strain input, with the respective experimental data presented in section 3.2 was considered satisfactory. These predictions were also consistent with the empirical law represented by eqn (2). The FE model was thus found an acceptable means of generating simulated databases for surrogate modelling.

\subsection{Surrogate model}

\subsubsection{Background}

The objective of the surrogate modelling is to replace an expensive function $f$ mapping a set of inputs $\mathbf{x}$ to a set of outputs $\mathbf{y}$ with a simpler approximate function $\hat{f}$ in order to reduce the computational cost of evaluating $\mathbf{y}$ for a new set $\mathbf{x}$. A surrogate model is based on a number of observations called training data; it can be considered as a multi-dimensional curve regression. In the present problem, $f$ is the FE model, set $\mathbf{x}$ comprises the plasticity parameters $\left(\sigma_{\mathrm{Y}}, \sigma_{1}, A\right.$, $n$ ) while $\mathbf{y}$ corresponds to the indentation set $(B, m)$. Thus, in the present case, the surrogate model has the form

$$
(B, m)=\hat{f}\left(\sigma_{Y}, \sigma_{1}, A, n\right)
$$

For this characterisation project, surrogate modelling was performed using the SUMO software [11], which is freely available for academic use, it is well documented [12] and it interfaces with MATLAB.

\subsubsection{Training data}

A random selection of fictitious values for the set $\left(\sigma_{Y}, \sigma_{1}, A, n\right)$ was generated using a relevant MATLAB script. The selection was subjected to the constraints: 
- $\sigma_{Y}<\sigma_{1}, \varepsilon_{Y}=\sigma_{Y} / E<\varepsilon_{1}=\left(\sigma_{1} / A\right)^{1 / n}$.

- $\varepsilon_{1}=\left(\sigma_{1} / A\right)^{1 / n}<0.02$ so that the polynomial portion of the $\sigma-\varepsilon$ curve is kept within realistic limits.

- The gradient of the polynomial at $\left(\sigma_{Y}, \varepsilon_{Y}\right)$ must be lower than the Young's modulus $(E=70 \mathrm{GPa})$.

- The gradient at $\left(\sigma_{1}, \varepsilon_{1}\right)$ must be lower than at $\left(\sigma_{Y}, \varepsilon_{Y}\right)$ since the gradient of the $\sigma-\varepsilon$ curve must decrease with strain.

A reasonably even spread of $95 \sigma-\varepsilon$ curves were produced. The same MATLAB script also compiled the FE input file for each fictitious alloy. The simulated indentation parameters $(B, m)$ were obtained by running the $\mathrm{FE}$ program for each $\left(\sigma_{Y}, \sigma_{1}, A, n\right)$ set and fitting eqn (2) to the output.

\subsubsection{Validation}

It was considered important to check whether the surrogate model returns a similar output to that produced by the FE model it replaces. For this purpose, ten additional $\left(\sigma_{Y}, \sigma_{1}, A, n\right)$ sets, not included in the training data, were randomly generated. Both the FE and the surrogate model were run with these input data and the corresponding outputs were compared. The load-indentation diagonal relations predicted by FE and SUMO were in excellent agreement. It was thus demonstrated that the surrogate model accurately recreates FE results.

\subsection{Optimisation}

\subsubsection{Objective function}

A numerical optimisation analysis is required for the solution of the inverse problem, that is, the determination of a material parameter set from a known indentation parameter set. The objective is to minimise an error function of the form

$$
\phi=\left(\frac{B-B_{\text {test }}}{\operatorname{range}(B)}\right)^{2}+\left(\frac{m-m_{\text {test }}}{\operatorname{range}(m)}\right)^{2}
$$

where $\phi$ is also known as the objective function, $B_{\text {test }}$ and $m_{\text {test }}$ are the indentation parameters of the test alloy whose elasto-plastic properties are sought. The variation of $B$ and $m$ with the elasto-plastic parameters within the optimisation process is provided by the surrogate model.

\subsubsection{Genetic algorithm}

The adopted optimisation tool in this characterisation project was a genetic algorithm (GA) package available in MATLAB. Genetic algorithms are so called because they mimic biological evolution. An initial population of potential solutions (the individuals) are created in the problem domain using a random number generator. The individuals' performance in the problem domain (fitness) is assessed by the objective function. Individuals are selected for reproduction according to a selection function; the best individuals have a higher probability of being selected. Each individual is encoded with a unique string (or genotype), just as genetic information is encoded in chromosomes in the natural world. Reproduction combines the parents' genes to create offspring. A proportion of 
the offspring may alternatively be created by random mutation of the parents' genes in an attempt to widen the search space and encourage genetic diversity. This is supposed to reduce the likelihood of early convergence to a sub-optimal solution. Only by decoding the offspring's genotype can their performance in the problem domain (phenotype) be assessed. The cycle then repeats to create successive generations. The offspring's performance is expected to improve with each generation, whilst less fit individuals are allowed to die out. When a pre-set termination criterion is met, the algorithm stops, for example, when a set number of generations have passed without improvement in the fitness of the population. Additional features may be incorporated such as migration between sub populations. This is supposed to encourage genetic diversity.

The MATLAB GA comes with various options for creation, selection, reproduction, mutation and migration functions.

\subsubsection{Selection of GA options}

GA options and functions were adopted through a process of repeated trials and gradual improvements. The initial population was seeded with individuals having similar indentation characteristics to those of the tested alloy. Seeding gives the GA a legitimate starting point and it was not found to narrow the search space or cause early convergence. The rest of the initial population were created between upper and lower bounds and adhering to the same constraints as described in section 4.2.2 for training data production. These constraints kept the population realistic.

The population size was set at 100 . The argument in favour of a large initial population is that it minimises the chance of not searching part of the problem domain. Populations larger than 100 were tested but no noticeable improvement was observed; the optimisation merely took longer.

A scaling function ranked individuals from best to worst based on their value of $\phi$. Selection was achieved using a roulette wheel approach, that is, parents were allocated a segment with area proportional to their expectation after being ranked. A random number generator was then used to simulate where the roulette wheel would stop. The best $10 \%$ of the population were guaranteed to survive into the next generation to preserve individuals with favourable genes.

Mutation was used to create $50 \%$ of all offspring in an attempt to encourage genetic diversity. It was found that the GA converged prematurely if using the default setting of $20 \%$. Reproduction was selected to be Heuristic which creates offspring that lie randomly on the line containing the two parents, nearer to the parent with the better fitness value, in the direction away from the parent with the worse fitness value. Migration between populations was not found to produce better solutions though this could be because the genetic diversity achieved by having a large population and high mutation fraction obscured any effects of migration.

\section{Results}

Three fictitious test alloys, chosen deliberately to cover a wide range of indentation behaviour, were used to assess the accuracy of the GA predictions. 
The characterisation predictions, along with the actual stress-strain behaviour for these alloys, are plotted for comparison in fig. 4. If this methodology is applied to the characterisation of a real component, the user would not have the benefit of knowing the actual stress-strain curves (solid lines in fig. 4) for comparison and thus no way of assessing the accuracy of the predictions. However, if the predictions in the figure are typical across the full range of alloy behaviour explored in this characterisation project then they provide some confidence in the reliability of the adopted methodology.

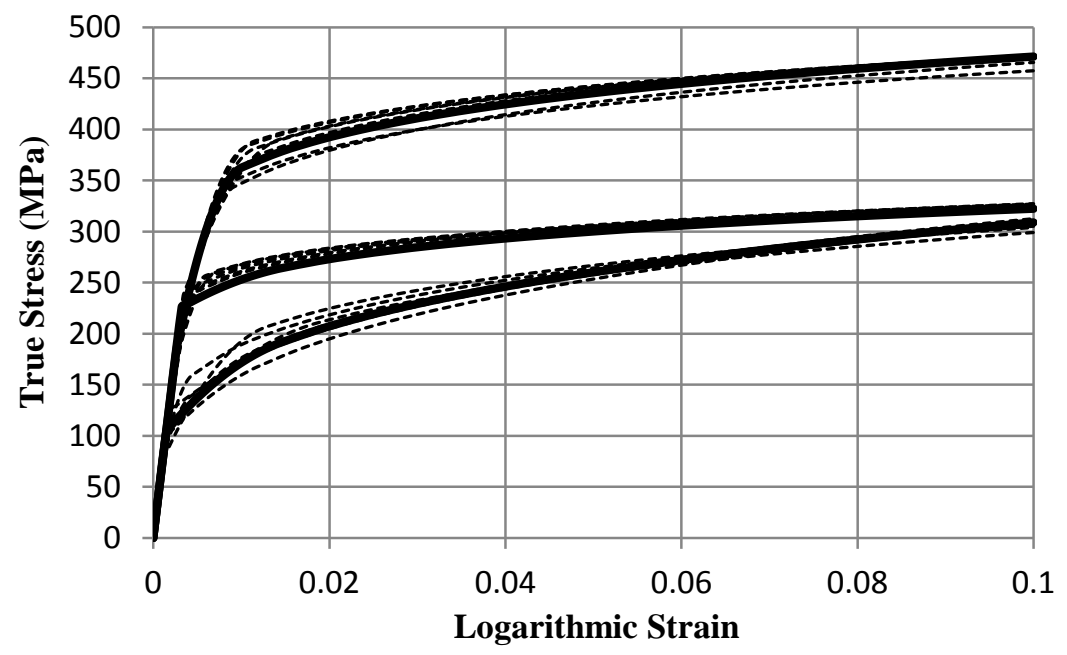

Figure 4: Characterisation predictions (dotted lines) plotted with the $\sigma-\varepsilon$ curves of the "tested" alloys (solid lines).

The GA did not arrive at the same prediction twice; this can be attributed to the nature of GAs which start with a population mostly comprising random guesses. The random initial population is different each time the algorithm runs so naturally the end results differ slightly. The predictions follow the general trend of the test alloys reasonably well, that is, there are three distinct groups of predicted curves in fig. 4, each clustered around that of the test alloy which they were intended to reproduce. It is reasonable to assume that if further runs were to be conducted, the GA solutions would be in the same range as those already observed; the nature of the GA is to kill off particularly wayward solutions. As for the accuracy of individual material parameter predictions, $\sigma_{Y}$ appears particularly difficult for the method to predict, sometimes underestimated by up to $50 \%$. Predictions for $\sigma_{1}$ and $A$ are much closer, reliably within $10 \%$ of the test alloy values, whilst $n$ is typically within $25 \%$ of the test alloy value.

As a check, the surrogate model was called manually with the GA's predictions after each optimisation was completed. The model confirmed that each GA solution was indeed an alloy with the same $B$ and $m$ as those of the test alloys, correct to four decimal places. This is the same precision that test alloy 
parameters were entered in the objective function. This could imply that multiple solutions exist and that each time the algorithm was run a new solution was discovered. However, it may simply mean that $B_{\text {test }}$ and $m_{\text {test }}$ were not specified with a great enough precision in the objective function for the algorithm to locate the global minimum.

As a further test, the GA was run with the correct solution seeded in the initial population. When given the test alloy parameters, the GA confirmed them as a solution. The minimum value of the fitness function in these cases was no less than when running the GA properly, that is, with random initial guesses. This supports the theory that the process, at this level of its development, generates multiple acceptable solutions.

\section{Conclusions}

In conclusion, the GA, combined with SUMO, is able to predict the general trend of an alloy's stress strain curve, but with room for improvement. It is worth noting in the results of fig. 4 that predictions for the test alloy in the middle of the meta-model range are more consistent with the target stress-strain curve than in the other two cases lying closer to range's edges. It may therefore be possible to enhance the effectiveness of the methodology by widening the range of material behaviour so that realistic alloy data always lie well within it.

The adopted method of selecting the training data may not have produced an even distribution of the independent variable set $\left(\sigma_{Y}, \sigma_{1}, A, n\right)$ within their ranges. This can be achieved by software developed for this purpose such as the Latin Hypercube sampling strategy [13], which is actually a feature of the employed SUMO toolbox.

Among the various elasto-plastic parameters, the largest discrepancy is noted in the prediction of the yield stress. In this respect, significant improvement could be achieved using Tabor's empirical relation between yield stress and Vickers hardness number [1] to seed the initial population with individuals having likely yield stresses.

There was significant difference between the indentation parameters $B$ and $m$ obtained from the experimental $P-\delta$ record and those resulting from the corresponding FE simulation. The apparent good agreement between experimental and simulated $P-\delta$ curves means that $B$ and $m$ are very sensitive to small variations in this relation. This issue could be addressed by having a denser set of experimental indentation data and further validation of the FE model. Improving the quality of the experimental output from both tensile and hardness tests would also contribute to removing this discrepancy. Specifying a greater precision on the values of $B$ and $m$ in the objective function would allow fitness to be assessed to a correspondingly greater precision which may aid the GA in locating the optimum solutions.

The accuracy with which ANSYS can predict indentation data influences the accuracy of the entire methodology. Testing of the surrogate model and optimisation stages has been conducted only using fictitious alloys; this has masked the problem to some extent. The methodology could only be applied to 
real alloys if the accuracy of the FE element simulations used to create training data is improved; this would enable a surrogate model to be generated that is capable of reliably predicting real indentation data from real elasto-plastic parameters.

\section{References}

[1] Tabor, D., The Hardness of Metals, Clarendon Press: Oxford, 1951.

[2] Bhattacharya, A.K. and Nix, W.D., Analysis of elastic and plasticdeformation associated with indentation testing of thin-films on substrates. International Journal of Solids and Structures, 24(12), pp. 1287-1298, 1988.

[3] Jayaraman, S., Hahn, G.T., Oliver, W.C. and Bastias, P.C., Determination of monotonic stress-strain curve of hard materials from ultra-low-load indentation tests. International Journal of Solids and Structures, 35(5-6), pp. 365-381, 1998.

[4] De Fazio, L., Syngellakis, S., Wood, R.J.K., Fugiuele, F.M. and Sciume, G., Nanoindentation of CVD diamond: comparison of an FE model with analytical and experimental data. Diamond and Related Materials, 10(3-7), pp. 765-769, 2001.

[5] Setiawan, R., Syngellakis, S. and Hill, M., A Metamodeling approach to mechanical characterization of anisotropic plates. Journal of Composite Materials, 43(21), pp. 2333-2349, 2009.

[6] Huber, N., Nix, W.D. and Gao, H., Identification of elastic-plastic material parameters from pyramidal indentation of thin films. Proceedings of the Royal Society of London Series A-Mathematical Physical and Engineering Sciences, 458, pp. 1593-1620, 2002.

[7] Muliana, A., Steward, R., Haj-Ali, R.M. and Saxena, A., Artificial neural network and finite element modeling of nanoindentation tests. Metallurgical and Materials Transactions A-Physical Metallurgy and Materials Science, 33(7), pp. 1939-1947, 2002.

[8] Liu, J., Christensen, S.W., Reed, P.A.S. and Syngellakis, S., Elastic-plastic characterisation of aluminium bearing alloys. Materials Science Forum, 396-4, pp. 1091-1096, 2002.

[9] ASTM, Metal Test Methods and Analytical Procedures, 2003.

[10] Nadai, A.I., Theory of Flow and Fracture of Solids, McGraw-Hill: New York, 1963.

[11] SUrrogate MOdelling (SUMO) Toolbox, http://www.sumowiki.intec.ugent. be/index.php/Main_Page, last accessed: April 2011.

[12] Gorissen, D., Crombecq, K., Couckuyt, I., Dhaene, T. and Demeester, P., A surrogate modeling and adaptive sampling toolbox for computer based design. Journal of Machine Learning Research, 11, pp. 2051-2055, 2010.

[13] Forrester, A., Sobester, A. and Keane, A., Engineering Design via Surrogate Modelling, Wiley: Chichester, UK, 2008. 